\title{
Software Defined Radio Architecture Contributions to Next Generation Space Communications
}

\author{
Thomas J. Kacpura \\ NASA Glenn Research Center \\ MS 54-1, 21000 Brookpark Road \\ Cleveland, OH 44135 \\ 216-433-6830 \\ thomas.j.kacpura@nasa.gov
}

\author{
Wesley M. Eddy \\ MTI Systems \\ at NASA Glenn Research Center \\ MS 54-1, 21000 Brookpark Road \\ Cleveland, OH 44135 \\ 216-433-6588 \\ wesley.m.eddy@nasa.gov
}

\author{
Carl R. Smith, John Liebetreu \\ General Dynamics Advanced \\ Information Systems \\ 8201 E. McDowell Road \\ Scottsdale, AZ 85257 \\ Carl.Smith@gd-ais.com \\ John.Liebetreu@gd-ais.com
}

\begin{abstract}
The structure of candidate next-generation integrated communication architectures for space communications and navigation address technologies, architectural attributes, mission services, and communications capabilities is improved by using software defined radios (SDRs). Evaluating lessons learned from development and operation of the early space SDRs on the NASA Space Communications and Navigation (SCaN) Testbed on the International Space Station (ISS) provide feedback for defining the communications architecture. An important attribute is leveraging SDR reconfigurability, which can be changes the way that operations are conducted.
\end{abstract}

\section{TABLE OF CONTENTS}

1. INTRODUCTION..............................1

2. SPACE COMMUNiCATIONS

ARCHITECTURE DRIVERS

3. SDR TECHNOLOGY AS A KEY

ARCHITECTURE ENABLER

4. IMPORTANT AREAS OF ONGOING PlatForm AND WAVEForm RESEARCH AND DEVELOPMENT........................6

5. SUMMARY AND CONCLUSIONS..............9

6. REFERENCES...............................9

7. BIOGRAPHY............................10

\section{INTRODUCTION}

Space communications architecture concepts, comprising the elements of the system, the interactions among them, and the principles that govern their development, are essential factors in developing National Aeronautics and Space Administration (NASA) future exploration and science missions. Accordingly, vital architectural attributes encompass flexibility, the extensibility to insert future capabilities, and to enable evolution to provide interoperability with other current and future systems.
Space communications architectures and technologies for this century must satisfy a growing set of requirements, including those for Earth sensing, collaborative observation missions, robotic scientific missions, human missions for exploration of the Moon and Mars where surface activities require supporting communications, and in-space observatories for observing the earth, as well as other star systems and the universe. An advanced, integrated, communications infrastructure will enable the reliable, multipoint, high-data-rate capabilities needed on demand to provide continuous, maximum coverage for areas of concentrated activity. Importantly, the cost/value proposition of the future architecture must be an integral part of its design; an affordable and sustainable architecture is indispensable within anticipated future budget environments.

Effective architecture design informs decision makers with insight into the capabilities needed to efficiently satisfy the demanding space-communication requirements of future missions and formulate appropriate requirements. A driving requirement for the architecture is the extensibility to address new requirements and provide low-cost on-ramps for new capabilities insertion, ensuring graceful growth as new functionality and new technologies are infused into the network infrastructure. In addition to extensibility, another key architectural attribute of the space communication equipment is interoperability with other NASA communications systems, as well as those communications and navigation systems operated by international space agencies and civilian and government agencies.

To support future science and exploration missions, the National Aeronautics and Space Administration (NASA) Space Communications and Navigation $(\mathrm{SCaN})$ program has defined a new space 
communications architecture and roadmap, including definitions of the network elements, the services supported between them, and a high-level development phasing approach [1, 2]. This architecture improves on many attributes of the legacy space communications systems that NASA uses, and includes upgrades and new systems that will increase flexibility and extensibility of the infrastructure in order to ensure interoperability with existing and future systems.

Software defined radio (SDR) technology plays a critical role in implementing the new space communications architecture. SDRs can be deployed in ground systems, user-terminals onboard spacecraft, data links on unmanned science missions, and in space-based relay systems. In support of commonality and reuse between these different SDRs, NASA has defined the Space Telecommunications Radio System (STRS) architecture ${ }^{1}$ which addresses the SDR platform and waveform interfaces, as well as the external interfaces to the platform and spacecraft communications systems. The STRS architecture supports a cost-effective path to building the different SDR platforms and waveform applications that will fit into NASA's overall space communications architecture.

At the same time, SDR technology also can have an impact on spacecraft architecture of onboard buses and the partitioning of functions between physical boxes on the spacecraft. New SDR platforms are powerful computing devices that can implement additional functionalities beyond just the radio frequency (RF) interfaces that legacy dedicated communications hardware handled. This paper references these devices as Advanced Space Adaptable Platforms (ASAP). ASAPs can consolidate functionality by providing additional resources reducing overall size, weight, and power requirements on the spacecraft bus. The ASAP and spacecraft bus architecture need to be aligned in terms of the interfaces used for uplink/downlink data and for management and control of the device. These interfaces are increasingly important as the uplink/downlink data rates are increasing, and the availability of other networkable components in the spacecraft avionics is increasing.

Communications and navigation system philosophies, technologies, architectural attributes, mission services, and communications capabilities are examined to understand how SDRs can form a key structural component to achieve integrated communication architectures for space communications and navigation services. Lessons learned were reviewed from the development of the NASA SCaN Testbed which is currently on the International Space Station and efforts were identified to keep the standard useful and up to date. As SDR technologies become more broadly used within the space community, advancements in the SDR operating environment, higher data rates, networking, and security can be exploited. The prospect of sustaining an architecture that has applicability to multiple radios and multiple markets can reduce costs and provide frequent technology infusion opportunities.

There are advantages of leveraging SDR technology and the STRS architecture for implementing critical elements of NASA's space communications infrastructure and also creating advantages for user and relay spacecraft architectures. Operational and development changes can be driven by the use of SDRs, and identifies key areas of future work for delivering the maximum value out of ongoing SDR platform development efforts.

This paper provides a brief overview of SDRs and space communication architecture. A description of space communication architecture key drivers is in Section 2. Section 3 describes elements of the SDR technology that impact the architecture. Section 4 describes ongoing research and technology developments that can advance the technology and bring new benefits. Section 5 provides a summary of the paper and conclusions.

\section{SPACE COMMUNICATIONS ARCHITECTURE DRIVERS}

The SCaN Program Commitment Agreement (PCA) requires that $\mathrm{SCaN}$ evolve "services in a manner consistent with a space architecture framework and mission requirements and pursue cooperation, collaboration, and cross-support with industry and other Government agencies, including international space agencies." The PCA assigns the SCaN Program responsibility for providing communications and navigation services (including systems engineering and planning) to user missions, and maintaining and evolving the SCaN architecture to effectively and efficiently meet user missions' present and future needs.

Three key requirements selected from the $\mathrm{SCaN}$ Level 2 Driving Requirements that directly motivate continued work on SDR technologies are: 
- SCaN shall implement a networked communication and navigation infrastructure across space

- SCaN's infrastructure shall evolve to provide the highest data rates feasible for both robotic and human exploration missions

- SCaN shall assure data communication protocols for space exploration missions are internationally interoperable

The first requirement for networking is motivated by the addition of multi-hop relay communications services using networking protocols and store-andforward techniques. This alleviates disconnections and high-delays present in interplanetary communications and other scenarios with incomplete or insufficient coverage for all users. The use of networking is also applicable to other relay spacecraft scenarios where traffic to/from multiple access links to user spacecraft is trunked to/from higherbandwidth links between the relay spacecraft and terrestrial infrastructure. This requirement will cause changes throughout the space communication systems. Existing earth and space-based elements do not generally support networking though networking technology is used extensively in the existing ground systems to move the space link bit streams and frame data within the terrestrial infrastructure. This requirement is addressable through deployment of SDR platforms that enable waveforms to support legacy interoperability and future upgrades enabling network interconnectivity.

The second requirement, higher data rates, addresses the need to serve science and exploration missions that collect high volumes of data from sensors and instrumentation and the need for increased downlink data rates to get this data on the ground. Data flows such as High Definition (HD) video and Synthetic Aperture Radar (SAR) data, even when compressed, result in a significant amount of traffic. The data rate requirements for a relay operating at higher layers of the protocols stack to support multiple users (i.e. not as a bent-pipe frequency-division system) also drives the system to supporting higher data rates on relay trunk links and cross-links where multiple user flows can be multiplexed together onto the same link. SDRs enable the flexibility and extensibility needed to implement and control this multiplexing functionality predicated that the platform has sufficient resource to implement the new waveforms. Likewise, the host vehicle must be able to support the increased data rates on its internal bus.
The third requirement, international protocol standards support, addresses the need to reduce the complex set of interface options supported across the Space Network (SN), Near Earth Network (NEN), and Deep Space Network (DSN). Because of the wide variety of legacy service interfaces, the architecture must have radios and data processing systems that are extremely flexible and reconfigurable to support early focus on legacy modes and future operations based heavily on international standard modes. The movement towards standards is not isolated to the RF airinterface, but also the protocols and interfaces between the radios and the other payloads on the bus. This points very naturally to a modular SDR technology, as multiple waveforms can be provisioned and configured/reconfigured according to current and future needs, and interchangeable interfaces allow an efficient delivery of needed hardware.

These requirements imply additional functionality beyond what traditional spacecraft radio systems have included. Networking is not typically implemented in the radio systems as they typically act only as a modem with no knowledge of protocols within the data streams. High data rates involving higher-order modulation (e.g. 8PSK) and advanced coding (e.g. Low Density Parity-Check Codes (LDPC) and Turbo Product block codes are not yet widely used in legacy user terminals and ground systems. International interoperability, through implementation of Consultative Committee for Space Data Systems (CCSDS) and other standards, can be assessed at various layers of a protocol stack. The CCSDS is a multi-national forum for the development of communications and data systems standards for spaceflights. This involves using modulation, coding, link framing, and network packetization or bundling protocols to meet $\mathrm{SCaN}$ and other providers' network service onramps.

The SCaN Testbed has three SDRs onboard the ISS [3], and the initial on-orbit waveforms have implemented very basic networking protocols and CCSDS framing. However, at this time, the avionics flight computer implements part of the networking and data framing functionality, and it is not fully contained in the SDR waveform firmware. For example, the avionics flight computer uses a SpaceWire point-to-point link between the radios. The GD-AIS S-band SDR is a compact, low-power transceiver and accepts a stream of fixed-length packets containing CCSDS Advanced Orbital Systems (AOS) transfer frames. The SDR adds the frame synchronization markers and channel coding. 
Likewise on receiving data, the GD-AIS SDR performs Viterbi decoding and frame synchronization, extracts the transfer frame data, and delivers the data stream to the avionics flight computer on the SpaceWire link. All encapsulated data is managed by the avionics flight computer.

Meeting the new SCaN requirements and deploying the new systems will have costs and involve multiple upgrades or new elements. This involves building an advanced, integrated, space communications infrastructure with reliable, multipoint, high data-rate capabilities available on-demand to missions. The communications infrastructure will provide continuous, maximum coverage for areas of concentrated space presence and activity. Ground system upgrades are also required to sustain and enhance existing services of the SN, NEN, and DSN. Because budgets for future space missions are finite, the cost/value proposition of the future architecture must be an integral part of its design. The effective use of SDRs and the advancement of a common SDR architecture, enabled by STRS, play a vital role in achieving this affordability vision.

These requirements are only part of the $\mathrm{SCaN}$ Program requirements and act as a high-level starting point for the program's efforts. SDR technology is extensible and efficient, satisfying these types of new or refined requirements and enable low-cost onramps for new capabilities insertion, ensuring graceful growth as new functionality and new technologies are infused into the communications infrastructure. Systems deployed during early increments in a multi-year infrastructure build-up can be software-upgraded to enable interoperability with systems deployed later in future increments.

\section{SDR TECHNOLOGY AS A KEY ARCHITECTURE ENABLER}

How does the flexibility of SDRs change the way communication architecture is specified? Current procurement process and communication systems specifications have engineers spending significant amounts of time analyzing the detailed specification of RF communications parameters as traditional RF systems are fixed at manufacturing. Using a modular SDR technology can reduce the upfront specifications to high-level parameters like RF bandwidth, RF output power, data traffic volume, etc. All digital parameters (modulation, error correcting codes, framing protocols, cryptography algorithms, etc.) can be managed later in the project and programmed into the SDR later in the project lifecycle, approaching launch. The ability to make changes to a digital signal is only limited by the processing abilities of the platform, and not by the initially deployed modulation types or coding combinations. The main constraints on this system's adaptability will be the installed RF "front-end" components and the digital interface to the SDR from the payloads.

Another aspect of leveraging reprogrammable digital components is that the system can be designed for inflight modifications and not frozen in the as-launched configuration. Changes can be facilitated by the SDRs throughout the mission life of the radio, from development through operations. This may be beneficial in order to support new standards and achieve greater commonality and interoperability across the set of missions and infrastructure. For instance, the NASA Space Network currently supports a number of modulation schemes that were implemented by missions but have become out of date. Since the missions are unable to upgrade and still need to be supported, it has become costly and complex to maintain both new and legacy modulations on the ground segment. With pervasive SDR technology, waveforms throughout the network can be updated to newer, more common, and higher performing standards. The ability to adapt throughout the life of the mission potentially reduces overall costs to the agency and simplifies system operation and maintenance.

Modular configurations also allow the platform to implement the ASAP concept and support multiple communications, navigation, or instrument applications (simultaneously or independently) and leveraging a high platform TRL and wider user base as components are reconfigured for different applications.

Examples of modules that can be integrated are:

- High accuracy GPS receiver

- Advanced communications ports (Ethernet, WiMAX, Wi-Fi, Optical)

- Drivers for servicing tools

- Gyro sensors for positioning

- Cameras for positioning and damage assessment

To address cost and interoperability, a modular architecture must be capable of supporting existing interfaces and adapting to new interface standards. The SCaN Testbed radios are three examples. They were developed for compliance with NASA STRS the SCaN Testbed supports multiple communication standards for interaction with a host computer platform. The testbed avionics flight computer 


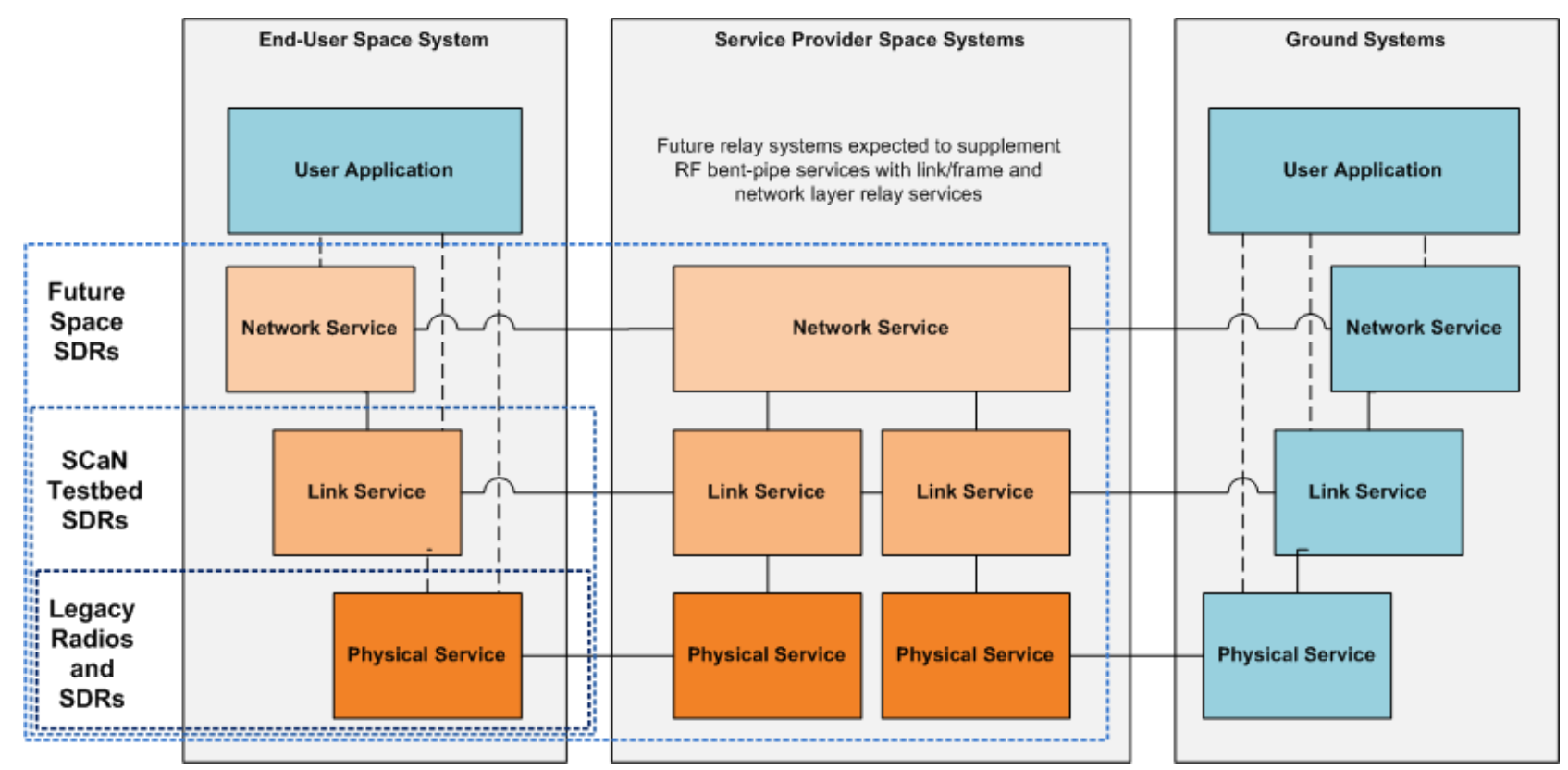

Figure 1 - Service On-Ramps Enabled By Future SDRs

communicates with the three radios via SpaceWire, MIL-STD-1553, and digital I/O lines. Over these physical interfaces, higher-level software interfaces can be implemented for data framing protocols, packet handling, platform monitoring and control protocols. Additional waveforms and software applications can be uploaded and dynamically support extension of the platform capabilities through additional cards.

SCaN's roadmap includes implementation of new service "on-ramps" at higher layers of the protocol stack. Legacy end-user radios and SDRs currently implement physical layer services (modulation and coding). Space-based relay systems such as the SN provide only RF bent-pipe relaying and do not include user services in the space segment. Only a physical layer on-ramp is supported. The SCaN Testbed SDRs currently support some link layer services, using CCSDS AOS transfer frames, and can support waveform extensions for additional AOSbased link service enhancements. For future spacebased relay services, the areas that the SDR waveforms would need to be extended are:

- Physical service support in waveforms for both forward and return link modulation and demodulation and for both sides of ranging and other services. Currently only forward link demodulation and return link modulations are typically implemented in spacebased SDR waveforms and ground radios implement the corresponding side. Because return link bandwidth is much higher, and higher-order modulations may be used, the high-rate receiver needed for a relay service may be beyond current space SDR platform capabilities.
Link service support for both forward and return services. Currently, SCaN Testbed SDRs support AOS framing and synchronization, but SCaN may support additional link layer interfaces with its operational services (e.g. Turbo Codes, TM, additional AOS features, and possibly Proximity-1). This primarily involves additional waveform code.

- Network service support, including the set of supporting protocols and features necessary to operate and manage a high-availability and high-rate relay system. This primarily involves additional waveform code and potentially uses more of the platform capability for networking built into the Commercial off the Shelf (COTS) operating systems that support STRS operating environments.

SDR technology provides a flexible way to start adding and deploying these features incrementally, which may save costs and simplify implementation and transition to the SCaN Integrated Network.

Additionally, as SCaN's integration efforts proceed and detailed implementation decisions are made about specific protocol versions, options, and feature an SDR-based approach to user terminals and relay systems will allow waveform adjustments that accommodate gradual enhancements. Further, there could be potential reuse between user, relay, and ground system waveforms, if developed to common standards like STRS. This will ease transition and save time and money in implementation and testing. 


\section{IMPORTANT AREAS OF ONGOING PLATFORM AND WAVEFORM RESEARCH AND DEVELOPMENT}

In this section, we describe several areas of ongoing work related to the SDR platforms and waveforms that we believe are important for meeting SCaN's long-term architectural needs using SDR technology. These are:

- Cost Reduction through Standards and Commonality

- $\quad$ ASIC Digital Resources

- Reprogrammable Digital Resources

- Advanced Digital/Analog Converters

- Onboard Interfaces

Cost Reduction through Standards and Commonality

Affordability is a fundamental aspect of all future space endeavors for NASA, commercial, and military assets. Interface commonality and standardization enables SDR vendors to reduce the repeated design adaptations to meet unique interface requirements for each contract.

The USAF GPS-III program is an example program that can be leveraged. It has begun the move towards a standard communication mechanism of employing an Internet Engineering Task Force (IETF) Internet Protocol version 6 (IPv6) protocol stack, which provides the significant advantages of an open standards-based approach. The completed GPS-III constellation will become a mesh network consisting of satellites interconnected via high-speed crosslinks to form a Wide Area Network (WAN). Each GPS-III satellite will have an IPv6 router supporting IETF standards-compliant Open Shortest Path First (OSPF) routing and the Simple Network Management Protocol (SNMP) for management. Each satellite also is capable of functioning as a high data rate gateway satellite when it has line-of-site visibility to an advanced antenna ground station. While the router has links associated with each modem onboard, it also supports links communicating to other network enabled payloads on the spacecraft, creating a Local Area Network (LAN). Thus, an authenticated user at any of the ground stations, payload operations control centers, or other authorized offices around the globe can communicate directly with payloads on any of the satellites without having to wait for a line-of-sight pass near the ground station.

These protocols in the GPS can be leveraged as a low-cost way to fill some of SCaN's future needs in the Space Internetworking capability. DTN and other enhancements can be implemented as higher level protocols.

Multiple advantages exist for NASA in using this capability:

- Using statistical multiplexing to reduce overall bandwidth trunking requirements by controlling routing and QoS parameters to manage the data flow through a relay constellation. This takes advantage of multiple gateways to share the space network bandwidth

- Using standard network management and routing protocols facilitates network growth and upgrades. For example, COTS largescale network management tools may be applicable rather than extensions of expensive custom one-off software packages

- Reducing cost by avoiding custom packet routing mechanisms, and instead encapsulating IPv6 over diverse physical interfaces. For example, encapsulating IPv6 over SpaceWire can enable direct routing between a high speed internal spacecraft LAN and the wider relay constellation and terrestrial WAN

- Reducing cost in integration of satellite payloads connected via standard based LAN versus proprietary interface protocols defined by individual bus providers

Internet Protocol (IP) and Delay Tolerant Networking (DTN) protocol suites are complex and include many optional features, performance enhancements, optimizations, and detailed deployment configuration decisions to be made. The exact protocol feature profiles to be used for space internetworking in the Integrated Network are subject to future definition and refinement, SDR is a flexible way to provide hardware implementations of prototype or early protocol feature profiles while the exact and final set are still being assessed between $\mathrm{SCaN}$ and user missions.

\section{ASIC Digital Resources}

Application Specific Integrated Circuits (ASICs) are still important components in the current SDR platforms. The Aeroflex UT90nHBD is a 90nm Complementary Metal-Oxide Semiconductor (CMOS) process fabricated using an IBM foundry. This ASIC family was developed by a team of industry leading technology and tool providers directed by the Defense Threat Reduction Agency (DTRA) of the United States government. The goal of this effort is to bring the performance and density 
advantages of commercially available 90nm ASIC technology to users requiring radiation hardness. This program started in 2011 and is expected to complete in early 2014. A recent program status briefing indicates the QML-V qualification efforts for the wafer fab technology, base library and assembly/packaging process are executing to plan. The final ASIC QML-V qualification report for the wire-bonded 472-pad LGA device is expected to be complete by December 2013. QML qualification for flip-chip packaging technology is on a similar schedule. Radiation testing is nearing completion per the specifications given below:

- $\mathrm{TID}>1 \mathrm{M} \operatorname{rad}(\mathrm{Si})$

- SEU rate of < 1x10-9 upset/bit-day

- SEL of $>120 \mathrm{MeV}^{*} \mathrm{~cm} 2 / \mathrm{mg}$

The UT90nHBD has the highest logic density and speed with the lowest power consumption of all the candidate ASIC technologies considered in a recent GD study. It also is available with an embedded SpaceWire core protocol handler. Given the current program status, it is expected that the UT90nHBD should easily reach Technology Readiness Level 6 by early 2015, which is a prototyping demonstration in a relevant end-to-end environment. Integration of the UT90nHBD and/or other high-performing radiationhardened ASIC components in non-reprogrammable portions of the SDR platform is an important area of ongoing work.

\section{Reprogrammable Digital Resources}

The space radiation environment can induce errors in processing that have until recently presented concerns on reliability and availability for reprogrammable hardware components. The mitigation has been to use ASIC or One-TimeProgrammable Field-Programmable Gate Arrays (FPGA) that take additional measures and resources to ensure operation, but do not alone support the full SDR reprogrammable hardware concept.

Xilinx has worked in a partnership with the Air Force Research Laboratory specifically for space applications to develop the Virtex-5QV (V5QV), a radiation hardened variant of the commercial Xilinx Virtex 5 FPGA. The space grade V5QV family was released in September of 2011 and has received inspace heritage through several SpaceCube missions.

The V5QV is manufactured using a $65 \mathrm{~nm}$ CMOS process and offers up to $1.5 \mathrm{M}$ equivalent ASIC gates. The V5QV offers an impressive array of resources including over 80,000 SEU-hardened flip-flops and 10k bits block Random Access Memory (RAM). Additionally the V5QV provides advanced DSP, clock generation and IO features. The radiation performance characteristics of the V5QV are provided below:

- $\quad \mathrm{TID}>1 \mathrm{M} \operatorname{rad}(\mathrm{Si})$

- $\quad$ SEU rate of $<3.8 \times 10-10$ upset/bit-day

- $\quad$ SEL of $>100 \mathrm{MeV}^{*} \mathrm{~cm} 2 / \mathrm{mg}$

Reprogrammable FPGA technology is Static Random Access Memory (SRAM) based and volatile, thus it requires external storage of the programming image. In spaceflight applications, this is generally accomplished using rad-hard non-volatile memory technologies such as Electrically Erasable Programmable Read-Only Memory (EEPROM), Computational Random Access Memory (CRAM) or Multibank Random Access Memory (MRAM). The programming image for a $\mathrm{V} 5 \mathrm{QV}$ is approximately 50 Mbits. Depending upon the storage technology employed, this could require multiple non-volatile memory devices.

In contrast, the SCaN Testbed GD SDR uses Virtex-2 technology, which was the best available at the time it was built, and for terrestrial applications Xilinx is selling the Virtex-7 family with a $28 \mathrm{~nm}$ design as its latest product line. Integration of the V5QV and other higher-performing radiation-hardened FPGA components that may be developed in the future is an important area of work in order to extend the processing capabilities of the reconfigurable digital logic portions of the future SDR platforms.

\section{Advanced Digital/Analog Converters}

The SDR “front end" portions include Analog-toDigital and Digital-to-Analog Converters (ADC/DAC), necessary to interface the digital processing components with the microwave components. For received signals, the ADC is the limiting component, and for transmitted signals, the DAC is relevant. Supporting higher data rates and flexibility in waveform configuration requires ADC/DAC components with sufficient:

- Sampling range in bandwidth, in order to process wideband signals or tune signals across a wider band

- Sampling rate in samples per second, in order to sample above the Nyquist rate for a given waveform configuration

- Sampling resolution in bits per sample in order to meet signal to noise ratio (SNR) specifications for the waveform 


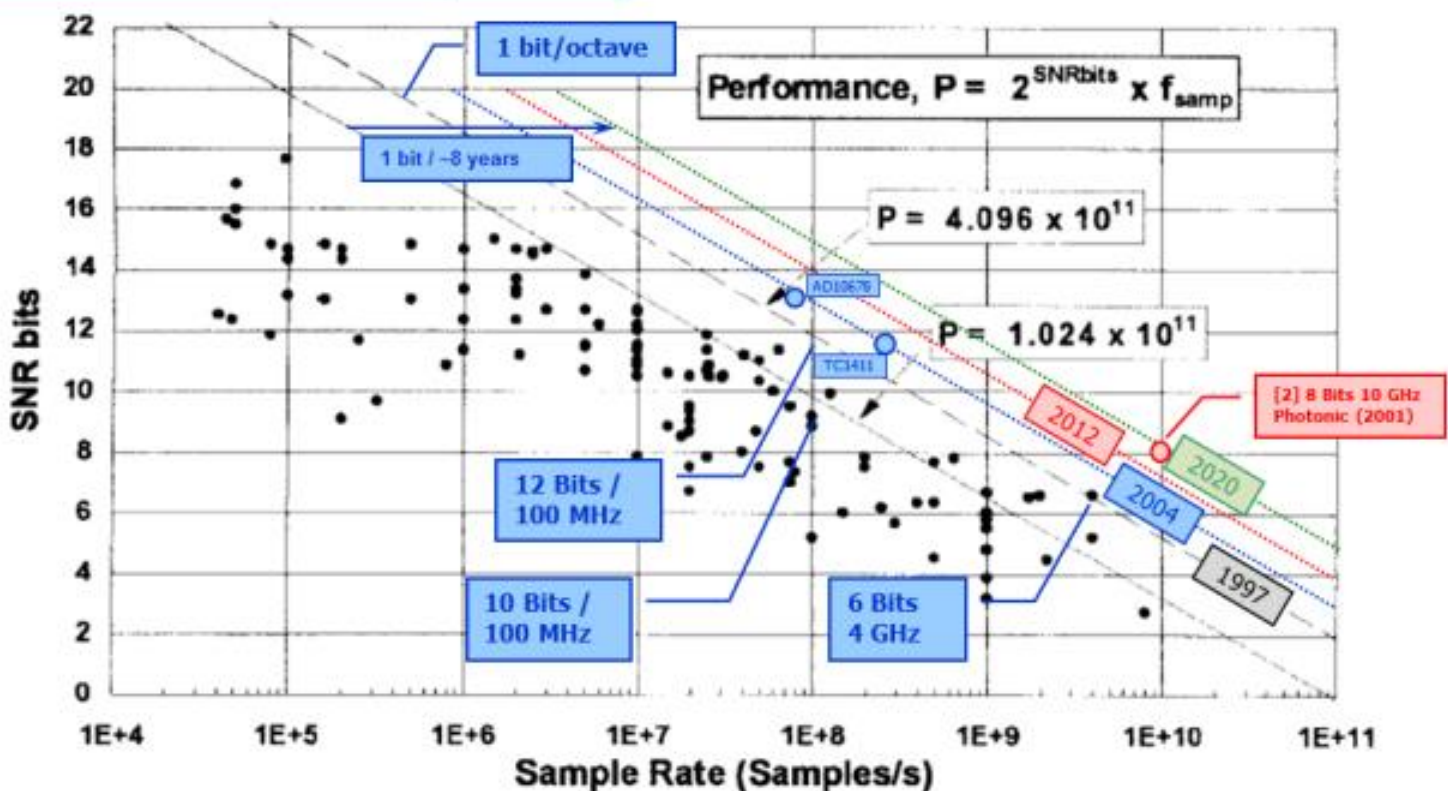

Figure 2 - ADC Technology Evolution

Figure 2 depicts the trend in state of the art performance for analog-to-digital converters, based on the performance metric reported by Walden [4]:

$$
\mathrm{P}=\text { 2SNR (bits) * fsamp. }
$$

Walden's performance metric trend lines are updated to show additional performance progress through 2004 and 2012, well as a projection for 2020, based on the observation that ADC performance improves by $\sim 1$ to 1.5 bits every 8 years. Several ADC performance benchmarks are also overlaid on the figure. The current state of the art is represented by the Analog Devices AD9467, 13 bits at $250 \mathrm{MHz}$.

An important focus for future work is to incorporate newer ADC/DAC components into the SDR front ends to accommodate SCaN's objective to support operation in multiple frequency bands $(\mathrm{S}, \mathrm{X}, \mathrm{Ku}, \mathrm{Ka}$, and potentially optical), high bandwidth signals, and low bit error rates.

\section{Onboard Interfaces}

For low-rate radio monitoring and control, as well as higher rate data links (e.g. hundreds of Mbps), there are multiple technology options (RS-422 serial, MILSTD-1553, Ethernet, SpaceWire, etc.) Separate interfaces are generally provided for monitor and control functions to the platform in contrast to the flow of user data through the radio. While the radio platforms may be modular enough to accommodate cards that support different physical interfaces, the formats for packets, frames, commands, and other data sent over the links requires a level of standardization that is not yet done. This would simplify and increase reusability to missions and relay spacecraft for the avionics flight computer other onboard systems that flow data through SDRs.

On the SCaN Testbed, for instance, of the three SDRs, two use MIL-STD-1553 for platform monitoring and control and the other uses SpaceWire

[5] All three SDRs have distinct command and telemetry packet formats and different concepts of how data should be formatted inside those packets. One SDR packs telemetry data as a set of defined fixed-length binary fields. Another SDR uses a configurable set of name-value pairs. For user data, all three also implement different designs, supporting either fixed-length full CCSDS transfer frames inside SpaceWire packets, only transfer frame bodies, or variable-length bitstream data flow over SpaceWire packets. Greater commonality in the user data formatting on this interface is necessary to simplify support in other onboard systems flowing data through future SDRs. The vendors chose the interfaces and protocols based upon their legacy products, which was expedient for them; however this resulted in a longer spacecraft integration time due to the lack of commonality and time to implement three different approaches.

Standard higher-layer interfaces to the platforms and waveforms are essential in order to reduce complexity in the relay management software for a space-based relay platform that may have multiple SDRs, potentially operating in multiple frequency bands. 


\section{SUMMARY AND CONCLUSION}

SDRs offer many advantages for implementing critical pieces of NASA's future space communications architecture, and they support the implementation and transition to an integrated network which offers new service onramps, enhanced flexibility, greater automation, and lower long-term operating and maintenance costs.

To maximize the potential of space SDRs for user terminals and space-based relays, a number of enhancements that should be made were identified. These are in the areas of:

- Cost Reduction through Standards and Commonality

- ASIC Digital Resources

- Reprogrammable Digital Resources

- Advanced Digital/Analog Converters

- Onboard Interfaces

The enhancements discussed in the paper are key improvement areas to provide future space SDRs that will be capable of meeting SCaN's goals. However, effectively using the features of SDRs also will require changes in the concept of operations for space communications services, which is beyond the current practices for RF bent-pipe and ground-based processing systems.

In order to take advantage of SDR reconfigurability in operations, a clear plan to thoroughly test the software updates needs to be prepared and executed so that the SDR performance does not result in any problems. The preferred approach is to test the new software on the ground on a similar version of the flight radio before changing the flight SDR. Offnominal operation of SDRs that provide mission critical communication for spacecraft commanding can result in losing control of a spacecraft. Fallback techniques to the previous software version to recover from nonperforming operating environment and waveform updates can be implemented which allow a graceful recovery. Failsafes for the platform are more difficult to implement, but also possible. For example while recovery techniques from upsets due to radiation or charged particles are effective, component hardware backups or a separate redundant system may be required to achieve mission reliability requirements.
Also, SDRs are used extensively in ground systems, though there has not been a large amount of portability between ground and space waveforms. The GD SDR onboard the SCaN Testbed uses an adaptation of the Bilateration Ranging Transponder System - Replacement (BRTS-R) waveform that was originally written for a terrestrial SDR, which shows that this reuse is viable. Because of the differences in processing platforms available for flight versus ground use, the amount of reuse may ultimately be limited, but further work on waveform portability between systems could save time and money in the long term.

NASA is not the only customer of these radios. Software defined radios are developed for other applications, and taking advantage of these developments promotes an architecture that is cost effective and sustainable. Developments in the following areas such as an updated operating environment, higher data rates, networking and security can be leveraged. The ability to sustain an architecture that uses radios for multiple markets can lower costs and keep new technology infused.

\section{REFERENCES}

[1] Liebrecht, P., Schier, J., Bhasin, K., Bibyk, I., Butler, M., Hudiburg, J., Tai, W., Shames, P., "NASA's Space Communications Integrated Architecture", Proceedings of SpaceOps 2010 Conference, AIAA, 25-30 April 2010, Huntsville, Alabama.

[2] Wallace Tai, Nate Wright, Mike Prior, and Kul Bhasin, "NASA Integrated Space Communications Network", American Institute. of Aeronautics and Astronautics SpaceOps Conference, Stockholm Sweden, 12-15 June 2012.

[3] Richard C. Reinhart, Thomas J. Kacpura, Sandra K. Johnson, James P. Lux, "NASA's Space Communications and Navigation Test Bed aboard ISS to Investigate Software Defined Radio On-board Networking and Navigation Technologies," IEEE Aerospace and Systems Magazine, Volume 28, Number 4, April 2013.

[4] R.H. Walden, "Analog to Digital Converter Survey and Analysis," IEEE Journal on Selected Areas in Communications, April 1999, pp.539-550.

[5] Thomas J. Kacpura, Denise M. Varga, "SCaN Testbed Software Development and Lessons Learned," 63rd International Astronautical Congress, Naples, Italy, 2012. 


\section{BIOGRAPHIES}

Thomas J. Kacpura received the B.E.E. degree in Electrical Engineering from Gannon University in 1985 and the M.Eng degree from Rensselaer Polytechnic Institute in 1986. He is a registered Professional Engineer in the State of Ohio. Since 1990, he has worked at the NASA Glenn Research Center. Activities include sounding rockets, Space Shuttle, and International Space Station microgravity experiment payloads. Also, development of space qualifiable phased array antennas and high data rate modems, and was a co-principal investigator for the Space Telecommunications Radio Standard (STRS) development. Thomas was the communications and SDR development lead for the SCaN Testbed SDRs and currently leads the advanced development communication systems area. He was part of team winning a $R \& D 100$ award for developing the first space Ka-band SDR. He is currently the project manager for delivering the next generation of NASA space communication compatibility test sets.

Wesley Eddy is Chief Technologist at MTI Systems, where he supports contracts with NASA and other agencies. He has an MSc degree in Computer Science from Ohio University, where he studied modifications to TCP congestion control. For NASA, he has worked on a number of space and aeronautics research projects, including the first demonstration DTN in space onboard the UK-DMC satellite and several studies involving space-based networking and protocol enhancements needed for the space environment. He has also worked as a systems engineer on NASA teams including the SCaNConstellation Integration Project (SCIP), the Space Network (SN) Ground Segment Sustainment (SGSS) project, the Altair lunar lander, and the SCaN Testbed. He is active in the Internet Engineering Task Force (IETF), where he co-chairs the Active Queue Management and Packet Scheduling (AQM) working group. In the past, he was an IETF Area Director and also co-chaired the IETF TCPM working group and the Internet Congestion Control Research Group (ICCRG)

Carl Smith has recently been a Software Technical Manager at General Dynamics AIS having worked with spaceborne communications including the Iridium satellite constellation, GPS receivers, and multiple NASA transponders. Recently, he has worked on the GPS-III network communications system, an Internet Protocolbased communications network for the GPS-III constellation based on General Dynamics Space-SDR technologies. His previous work at General Dynamics includes NASA CoNNeCT STRS Software Defined Radio; $M$-code enabled GPS receiver; and Orion CEV S-Band Transponder. He holds a BSEE from Polytechnic University and a MSEE from Drexel University. Carl has recently joined the engineering team at Fluidic Energy.

John Liebetreu, PhD, is a Distinguished Member of the Technical Staff at General Dynamics AIS Integrated Space Systems Division in Scottsdale, Arizona, where he works on space-borne communications and navigation systems. Dr. Liebetreu has worked on all aspects of advanced communications systems for over 25 years, beginning with NASA's ACTS (Advanced Communications Technology) program in the early 1980's. Recently he worked as the lead systems engineer with personnel from the NASA Glenn Research Center to develop the Space Telecommunications Radio System Architecture for Space-SDRs. He worked as the lead communications systems architect for General Dynamics on NASA's CONNECT program, a SDR path-finding program for future NASA space reconfigurable transceivers, and leads activities within General Dynamics for applying software defined radio technologies to space tracking, telemetry, navigation, and control applications. Dr. Liebetreu has participated in numerous standards development activities, including the SDR Forum Space Working Group, where he led the hardware architecture review task group for the NASA STRS Architecture, and the IEEE 802.16 Working Group for broadband wireless standardization, where he served as the PHY Task Group Chair. He earned a BSEE from Michigan Technological University, an MSE from Arizona State University, and a PhD from University of Missouri-Rolla. 